\title{
Décadrages Décadrages
}

cinéma, à travers champs Cinéma, à travers champs

$12 \mid 2008$

Fredi M. Murer

\section{Allégories de « La Vie telle que nous la connaissons » : le regard ethnographique dans les fictions de Fredi M. Murer}

\section{Marcy Goldberg}

Traducteur : Pierre-Emmanuel Jaques

\section{OpenEdition}

\section{Journals}

Édition électronique

URL : http://journals.openedition.org/decadrages/370

DOI : $10.4000 /$ decadrages. 370

ISSN : 2297-5977

\section{Éditeur}

Association Décadrages

\section{Édition imprimée}

Date de publication : 10 avril 2008

Pagination : 54-61

ISBN : 978-2-9700582-7-4

ISSN : 2235-7823

Référence électronique

Marcy Goldberg, « Allégories de « La Vie telle que nous la connaissons » : le regard ethnographique dans les fictions de Fredi M. Murer », Décadrages [En ligne], 12 | 2008, mis en ligne le 05 février 2014. consulté le 10 décembre 2020. URL : http://journals.openedition.org/decadrages/370 ; DOI : https:// doi.org/10.4000/decadrages.370 


\section{la connaissons ": le regard ethnographique}

$\mathbf{1}$ Traduit de l'anglais par Pierre-Emmanuel Jaques.
2 Voir par exemple ses déclarations dans la brochure Fredi M. Murer - The Handwriting of the Senses (Pro Helvetia 1998) ou dans l'interview d'Irene Genhart qui figure en ligne dans la série Cinéportrait à: www.swissfilms.ch

3 "Fredi Murer hat immer betont, Höhenfeuer könne überall zwischen Island und Japan spielen, es sei inm aber auch immer klar gewesen, dass dieser Film mit ethnografischer Präzision in Szene gesetzt werden müsse, und so sei er eben wieder in der Innerschweiz, im Kanton Uri gelandet, seiner eigenen Heimat." Peter Purtschert, "Das Phänomen Höhenfeuer", dans Cinema 46: Heimspiele - Film in der Schweiz seit 1984, Chronos Verlag, Zurich, 2001, p. 110.

\section{dans les fictions de Fredi M. Murer ${ }^{1}$}

Tous les films de fiction de Fredi M. Murer sont construits comme des contes ou des récits de légende: Höhenfeuer (1985) s'apparente à une saga alpine et mythique, Vitus (2006) à un conte de fée moderne, Vollmond (1998) à un récit allégorique, Grauzone (1979) à une fable à connotation politique, 2069 (1969) à un récit de science-fiction dystopique. En outre, ils sont remplis d'observations pointues, voire de critiques de la vie quotidienne dans la Suisse contemporaine, le pays où ils sont tous manifestement situés. Dans plusieurs interviews, Murer lui-même a souvent évoqué le rapport d'amour-haine qu'il entretient avec ce pays où il est né et qui est à l'origine de ses films ${ }^{2}$. Höhenfeuer pourrait ainsi se situer n'importe où, en Islande ou au Japon, mais Murer a finalement choisi de le tourner en Suisse, dans son propre canton d'Uri, parce qu'il était convaincu que l'universalité de l'histoire devait être ancrée dans des détails précis de la vie quotidienne, dans une langue et des gestes particuliers, éléments qu'il ne pouvait reproduire avec fidélité qu'en partant de sa propre culture ${ }^{3}$. Le rôle de Murer en tant qu'ethnographe de sa propre société s'appuie sur sa pratique de documentariste, en particulier sur son chef d'œuvre Wir Bergler in den Bergen sind eigentlich nicht schuld, dass wir da sind (1974) consacré à la paysannerie de montagne - un film fait "avec» et non "sur» les habitants du Canton d’Uri comme l'indique le générique d'ouverture.

En tant que pratiquant d'une «Binnenethnologie» - une recherche ethnographique dans son propre pays - Murer est simultanément dans et hors de cette culture: il en est issu, mais préfère s'en extraire et se placer en marge pour en devenir un observateur attentif. Le point de vue de ses films n'est pas explicitement politique ni lié à un système idéologique précis, mais témoigne d'un scepticisme bienvenu. Dans une interview radiophonique menée par Charles Clerc en 2004, Murer décrivait son attitude politique de jeune adulte vivant alors en Suisse 
dans les années précédant 1968 comme globalement non-conformiste: il n'était favorable à aucun mouvement en particulier, mais s'affirmait résolument "contre» la mouvance dominante 4 .

C'est peut-être pour cette raison que le regard critique développé dans ses films diffère de celui des autres cinéastes de sa génération. Les films de Murer se distinguent notamment de ceux du «nouveau cinéma suisse» des années 1960 et 1970 dans leur traitement plus nuancé de la nature et du paysage. De nombreux films de cette période constituent un véritable règlement de compte avec les représentations idéalisées des montagnes suisses, typiques des générations précédentes, particulièrement avec les images patriotiques des Alpes issues de la «défense nationale spirituelle» de l'époque de la seconde guerre mondiale ${ }^{5}$. Pour nommer deux exemples représentatifs : le documentaire de Kurt Gloor Die Landschaftsgärtner (1969) et le film de fiction d'Yves Yersin Les petites fugues (1979) rompent avec le mythe du fermier héroïque, décrivant la vie rurale comme monotone, difficile et menacée par la pauvreté. Dans une scène maintenant classique de la déconstruction de l'iconographie alpine, le vieux paysan Pipe, le protagoniste principal du film de Yersin, dépense une part importante de sa rente vieillesse dans un voyage en hélicoptère sur le Cervin bien aimé, pour découvrir qu'il ne s'agit en fait que d'un amas de pierre («il n'y a que des cailloux»). Mais c'est surtout Alain Tanner qui a pratiqué une critique systématique du paysage dans ses films, analysée en profondeur par Maria Tortajada ${ }^{6}$. Comme elle le montre bien, Tanner ne rejette pas unilatéralement le beau paysage, mais il questionne la manière dont il a été instrumentalisé.

Pour Murer, au contraire, le paysage et la nature représentent une force spirituelle, quasi surnaturelle, qui domine l'être humain en dépit

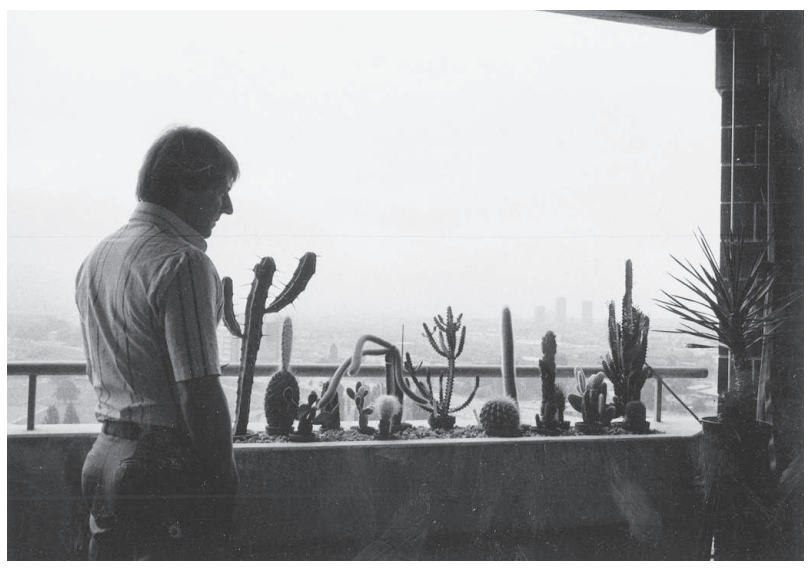

4 Emission "Music für einen Gast" diffusée sur la DRS 2 le dimanche 26 septembre 2004.

5 Voir Yvonne Zimmermann, "Die Berge aus Schweizer Sicht - ein Streifzug durch den Schweizer Film", dans Alexandra Schneider (éd.), Bollywood: Das indische Kino und die Schweiz, Zurich, Ed. Museum für Gestaltung, 2002, pp. 124-133.

6 Maria Tortajada, "Cinéma suisse: comment échapper au paysage narcissique?", dans Marc-Olivier Gonseth, Jacques Hainard, Roland Kaehr (éd.), Derrière les images, Musée d'Ethnographie , Neuchâtel, 1998. 
7 L'interview, non publiée, de Pio Corradi a été menée par Thomas Schärer dans le cadre du projet Cinememoire - Eine Oral History des Schweizer Films (2007-2009), soutenu par le FNS/SNF. Je le remercie pour m'avoir communiqué les résultats de cette recherche.

$\mathbf{8} \mathrm{La}$ bande sonore joue toujours un rôle important dans les films de Murer. Cette question du son, et notamment sa longue collaboration avec le compositeur Mario Beretta, sont des thèmes qui mériteraient une étude particulière en soi.

9 Martin Schlappner et Martin Schaub, Cinéma Suisse. Regards critiques 1896-1987, Bâle, Stroemfeld / Roter Stern, 1987 (traduit de: Vergangenheit und Gegenwart des Schweizer Films 1896-1987), pp. 92, 172-173. de ses efforts pour la maîtriser. Dans ses films, les pouvoirs thérapeutiques de la nature contrastent avec la stérilité des paysages urbains et modernes et ses personnages s'évadent souvent dans la nature pour retrouver un certain équilibre. Dans Grauzone, le technicien de surveillance Alfred se sent attiré par la forêt; dans Vollmond, le policier tracassé s'adonne à la pêche dans des rivières de montagne; dans Vitus, le jeune génie tourmenté trouve ses rares moments de bonheur à la campagne, en compagnie de son grand-père.

C'est surtout dans Höhenfeuer que les paysages intacts de la montagne forment un cadre puissant pour cette histoire d'inceste et de parricide. Les forces élémentaires de la nature - le vent, le soleil, la pluie - soulignent la dimension allégorique des événements dépeints, au-delà des détails, éminemment réalistes, de la vie quotidienne de la famille de montagnards. En même temps, Murer a été extrêmement attentif à ne pas reproduire les clichés de la "belle montagne»: dans une interview avec Thomas Schärer, le directeur de la photographie Pio Corradi décrit comment le réalisateur lui interdisait de cadrer en tableaux les majestueux sommets alpins, mais insistait pour qu'il les découpe en de multiples fragments ou qu'il les ampute de leur sommet ${ }^{7}$. En même temps, la piste sonore, qui comprend des compositions musicales de Mario Beretta et des sons naturels de vent, d'eau, d'animaux et d'insectes intensifie le caractère spirituel et surnaturel des scènes 8 . Avec cette juxtaposition d'images et de sons, Höhenfeuer représente le paysage de montagne de manière réaliste, mais sans lui dénier sa beauté et son pouvoir élémentaire - on serait presque tenté de dire mystique ou magique.

Comme Martin Schaub l'a bien noté, Höhenfeuer, en dépit de son cadre rural et de son style poétique et allégorique, traite de thèmes caractéristiques du "nouveau cinéma suisse» - tel que le rejet de la société dominante, le retour à la nature, le conflit père-fils 9 . En fait, en donnant à cette histoire les apparences d'une légende alpine sans âge, Murer a pu introduire des éléments plus radicaux comme l'histoire d'amour entre frère et sœur et le meurtre - au sens littéral du terme - du père dominateur: éléments qui auraient été plus difficiles à accepter dans un décor moderne et urbain, mais qui reflètent néanmoins la société réprimée et répressive que le cinéaste cherchait à critiquer.

Le héros sans nom de Höhenfeuer, un adolescent sourd et muet appelé simplement "der Bub» (le garçon) incarne un motif que l'on retrouve dans toute l'œuvre de Murer: l'innocence et l'intégrité de l'enfance avant que celle-ci ne soit corrompue par la civilisation. Cette vision quasi rousseauiste se retrouve dans tous les films de fiction de Murer. De même, les héros de ses films plus récents Vollmond et Vitus sont des 
enfants non-conformistes en tout genre. Mais peut-être la représentation la plus radicale de ce conflit se trouve-t-elle dans 2069 oder, wo sich Futurologen und Archäeologen gute Nacht sagen, le premier film de fiction de Murer, qui constitue le dernier épisode de Swissmade, à côté de ceux réalisés par Yves Yersin et Fritz E. Maeder.

2069 est la vision d'un futur dystopique, dans lequel une société techniciste, soumise au contrôle totalitaire de l'ordinateur, est contestée seulement par quelques rebelles à moitié sauvages réfugiés dans la nature - un souvenir du Meilleur des mondes d'Aldous Huxley. Outre les décors futuristes de H. R. Giger, Murer - comme Jean-Luc Godard dans Alphaville (1965) - use de lieux suisses réels et d'une architecture contemporaine pour construire cette dystopie imaginaire de l'année 2069, insistant ainsi sur les racines présentes de ce futur cauchemardesque. La liste des "outsiders» qui ne s'insèrent pas dans cette société du contrôle gérée par le soi-disant "Brain-Center» comporte "des poètes, des naturistes, des criminels, des anarchistes, des rêveurs, des fous, des intellectuels et des scientifiques». Et cependant, certains de ces outsiders semblent être euxmêmes des caricatures de figures tout helvétiques: ainsi le gardien qui "s'est tenu sur la frontière depuis 1291 avec son arme non-chargée» et qui prétend être "le dernier Suisse défendant la liberté et la démocratie» bien qu'il "ne possède pas un seul mètre de ce pays". En fin de compte, on pourrait dire que le point de vue de Murer correspond à celui de la créature extraterrestre venue d'une "Entwicklungsplanet» (planète en voie de développement) qui étudie la société de 2069 et dont le point de vue subjectif est celui qu'adopte le récit - notamment par le fait que la caméra est rivée sur la tête de la créature. Une fois encore, le cinéaste Murer ne prend pas parti dans un conflit idéologique, mais joue le rôle

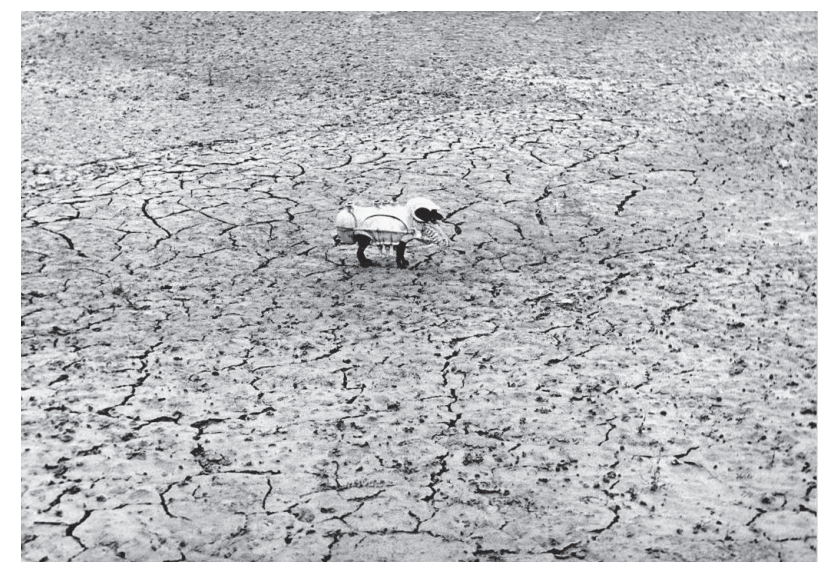


d'un ethnographe analysant les contradictions de sa propre société: un patriotisme réactionnaire appelant au retour à la terre face à un "progrès» technocratique déshumanisant.

Les personnages de 2069 parlent de "rupture» (Umbruch) pour désigner le moment où la "vieille démocratie partisane» a été remplacée par la soi-disant "démocratie plus humaine» de la bureaucratie soumise au contrôle totalitaire de l'ordinateur. Le thème de la rupture, de l'événement qui marque, fractionne, modifie le mode de vie se retrouve d'une manière ou d'une autre dans tous les films de fiction de Murer. Il joue un rôle clé dans la structure narrative qui évoque celle du conte de fée en prenant comme point de départ la question "Que se passerait-il si...?». Comme le "Violent Unexplained Event» (événement violent et mystérieux) qui change "Life As We Know It» (la vie telle que nous la connaissons) dans la parodie de documentaire The Falls (1980) de Peter Greenaway, l'idée d'une rupture dramatique permet à Murer de combiner hyperbole surréelle et vraie critique sociale. Dans Grauzone, tourné dans un noir et blanc onirique, la rupture est causée par le développement d'une mystérieuse épidémie de dépression et de souffrances ataviques que le gouvernement s'efforce de dissimuler au reste de la population. En plus de la dissimulation, de la surveillance et de l'hypocrisie pratiquées par l'Etat, Murer trouve aussi l'occasion de faire la satire du fonctionnement des médias, qui ne font que retranscrire de manière sensationnaliste les événements sans questionner la version officielle du gouvernement. La vérité est transmise par la mystérieuse station pirate Radio Eisberg qui décrit ainsi les symptômes de l'épidémie:

«Ressentez-vous soudain le besoin de gagner l'air libre? Vous sentez-vous attiré par les lieux de votre passé? Aimez-vous les levers de soleil ? Sombrez-vous sans raison apparente dans une profonde tristesse? Avez-vous le sentiment que votre peau devient toujours plus fine? Que vous ne vous souvenez plus de rien? Refusez-vous la possibilité de faire enfin quelque chose de votre propre volonté? Sombrez-vous de manière inattendue dans un sommeil sans rêve?» Les symptômes de l'épidémie sont typiquement rousseauistes et/ou romantiques: le désir de retour à la nature et à sa propre enfance, pour faire renaître des émotions éteintes depuis longtemps par le conformisme et la vie en société. La "zone grise» de la cité bétonnée entre en contradiction avec les "espaces verts» de la nature - un message qui pourrait paraître simpliste à première vue.

Mais la vision que développe Murer en 1979 d'un mécontentement larvé au sein d'une population manipulée et contrôlée par son gouvernement s'avéra prophétique, anticipant aussi bien les «événements» du 
début des années 1980 caractérisés par la révolte de la jeunesse que l'«affaire des fiches", le scandale de l'Etat espionnant ses propres citoyens qui éclata au début des années 1990. Le nom Radio Eisberg fait allusion à la métaphore alors très répandue de la Suisse comme un iceberg, une île glacée et isolée - une image qui devint particulièrement populaire parmi les jeunes activistes des années 1980 et qui est évoquée dans de nombreux films traitant de cette période, dont Züri brännt (1980) du collectif Videoladen et E nachtlang Fü̈̈rland (1981) de Clemens Klopfenstein et Remo Legnazzi. En accord avec la rhétorique destructrice (mais ludique?) de cette époque et en reprenant des slogans tels que "Rasez la mer que l'on voit la mer", Grauzone se conclut sur la vision d'un bâtiment qui explose. C'est l'ancien lieu de travail du protagoniste Alfred M. - un alter ego de Fredi Murer? - ce spécialiste de l'écoute clandestine qui vient de révéler à ses collègues la présence d'un système de surveillance au sein de l'entreprise qu'il vient de quitter. La conclusion du film, qui semble lier libération et destruction, présente la plus grande des "ruptures", laissant en effet l'avenir d'Alfred ouvert et incertain. Cependant cette ambiguiité correspond parfaitement au ton surréel du film. Comme dans Höhenfeuer, en combinant l'observation attentive de détails de la vie quotidienne, notamment une architecture et des décors authentiques, à une structure narrative liée au fantastique, Murer peut ainsi placer ses personnages dans des situations extrêmes de manière à offrir au monde hors du film un miroir critique.

L'irrationnel au service d'une critique rationnelle: cette tactique sert aussi de moteur dans Vollmond. Là encore l'histoire commence avec un "Et que se passerait-il si...?": la disparition de douze enfants une nuit de pleine lune. Ce que les adultes - et en particulier les autorités - prennent pour un enlèvement de masse se révèle être en fait une révolte des enfants en faveur d'un plus grand respect pour l'enfance et l'environnement, ainsi que d'une plus grande tolérance entre les gens. Le slogan des enfants, qui détourne l'idéologie chrétienne, est: "Wir wollen die Erde auf Erden» (Nous voulons la terre sur la terre). Malheureusement, la crise provoquée par l'action des enfants mène les adultes aux pires comportements, ceux-ci faisant preuve plus que jamais d'intolérance, d'agressivité et de manque de compréhension. A la fin du film, lors de la nuit de pleine lune suivante, ce sont cette fois douze fois douze enfants qui disparaissent.

Le caractère allégorique de ce récit permet à Murer de traiter un grand nombre de thèmes et problèmes: la destruction de l'environnement, l'incompétence des autorités, l'hypocrisie des adultes qui ont perdu le contact avec leurs enfants et avec la capacité d'émerveillement 
Vollmond (1998)

10 Le scénario de Vitus a été coécrit par Peter Luisi, un jeune cinéaste dont le premier long métrage Verflixt verliebt (2004) est une satire mordante de la production cinématographique indépendante en Suisse.

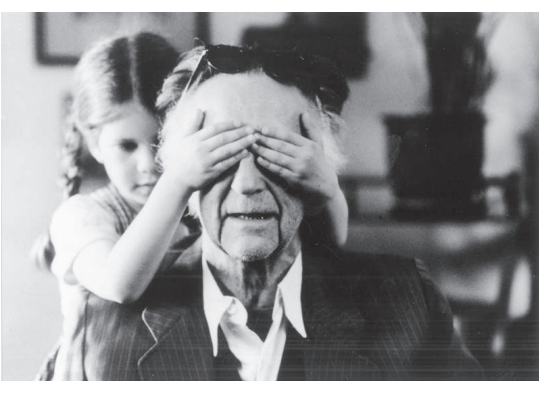

qu'ils avaient eux-mêmes en tant qu'enfants, et - une fois encore - les effets d'un appareil médiatique parasitaire qui exploite la catastrophe sans contribuer à la résoudre. Le film fait des allusions répétées aux structures politiques et sociales propres à la Suisse, et insiste sur le fait que les enfants disparus proviennent de toutes les régions linguistiques et géographiques du pays. Le monde que présente Vollmond correspond à la vision qu'a Murer de la Suisse contemporaine, en version modèle réduit. Le caractère fantaisiste de l'histoire est contrebalancé de manière critique par la précision des détails observés. Et cela dès les plans d'ouverture du film: une séquence sous-marine dans laquelle on aperçoit le fond du lac de Zurich jonché de déchets produits par la société de consommation, dont plusieurs appareils de télévision.

Vitus, le dernier film de Murer à cette date, marque un tournant par rapport à ses œuvres précédentes dans la mesure où le film est moins explicitement politique et volontairement plus divertissant, l'ironie cédant la place au burlesque, le détail ethnographique à l'exagération comique10. Il y a beaucoup moins de références explicites à la Suisse - au-delà de l'usage des lieux de tournage et des dialectes alémaniques et les éléments liés au commerce et à la finance servent avant tout à faire avancer l'intrigue. Thématiquement, toutefois, le film revient à l'une des principales préoccupations de Murer: l'enfant sensible inadapté dans une société autoritaire et conformiste. Et pour ce faire, Murer se sert de plusieurs éléments du conte fantastique pour faire mieux ressortir son argumentaire. Son héros, Vitus, est un enfant prodige: un génie musical avec un QI hors norme, qui est aussi doué pour jouer à la bourse qu'au piano. Le père de l'enfant est un inventeur naïf et distrait genre "savant fou", sa mère anglaise une «stage mother» excessivement ambitieuse aux allures de sorcière de conte de fée. Vitus peut tout faire - sauf ressembler aux autres enfants et se faire des amis. Son premier acte de rupture consiste en la mise en scène de son accident, qui lui permet de prétendre qu'une blessure à la tête l'a privé de ses talents 
musicaux et intellectuels. Mais son dernier acte de rébellion, encore plus métaphorique, se déroule à la fin du film quand il s'envole littéralement dans l'avion qu'il a acheté à son grand-père avec de l'argent gagné en bourse. Il a renoncé à faire semblant d'être "normal» et est prêt maintenant à poursuivre la carrière à laquelle il était destiné dès sa naissance : pianiste de concert.

Comparée aux fins radicales de ses films précédents - mort, destruction ou disparition - le dénouement de Vitus, qui se clôt sur les applaudissements du concert, est un happy ending qui semble suspect. Mais c'est le personnage du jeune Vitus qui reste le plus troublant, bien après la fin du film : le petit bonhomme habillé d'un smoking et dont l'intelligence et les talents lui ont causé de telles souffrances; le garçon à la sensibilité d'enfant et d'une intelligence plus grande que celle de la plupart des adultes. En appliquant au film un regard formé à la vision des films précédents de Murer, il est très difficile d'accepter cette conclusion "au premier degré». Le personnage de Vitus, ses difficultés, et même ses succès invraisemblables en musique et à la bourse, font référence finalement à "Life As We Know It» pour reprendre l'expression de Greenaway. Ici aussi, Murer a usé d'une grande fantaisie et de l'exagération pour soulever des questions qui renvoient le spectateur au monde réel. Et si ce monde est moins explicitement suisse que dans ses films précédents, il y a peut-être une explication positive: l'iceberg n'aurait-il pas commencé à fondre après tout? 